\title{
Dynamics of the Shape of the Electric Arc and Associated Magnetogasdynamic Flows Arising from the Opening of Initially Closed Electrodes
}

\author{
A.P. Glinov, A.P. Golovin, P.V. Kozlov, K.V. Shaleev \\ Institute of Mechanics, Lomonosov Moscow State University, \\ Moscow, 119192, Russia \\ krestytroitsk@mail.ru
}

\begin{abstract}
Investigations have been carried out of an electric arc discharge in atmospheric air at atmospheric pressure, both between commensurable electrodes and massive anodes many times larger than the cathode. The main emphasis is placed on an experimental study of vertical arcs initiated by opening the electrodes by conducting high-speed video recording of the discharge and synchronously taking waveforms of the current and voltage of the arc column. It is shown that the flow of electric current and flows of the interelectrode medium substantially depends on the opening speed of the electrodes and the size and shape of the anodes.
\end{abstract}

Keywords: electric arc discharge, arc initiation, discharge instability, arc stabilization.

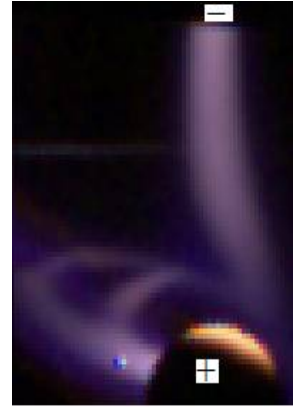

$a$

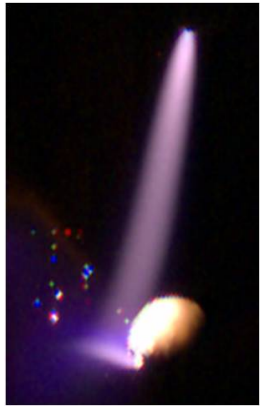

$b$

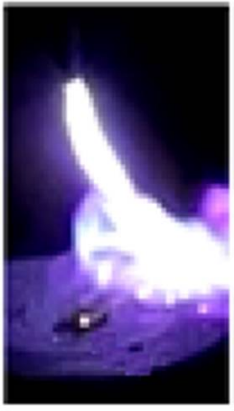

$c$

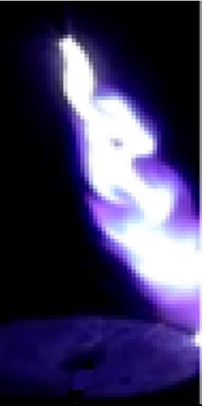

$d$

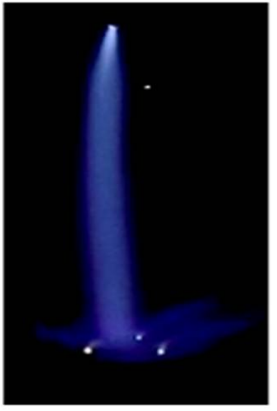

$e$

Video frames of an arc discharge initiated by opening previously closed electrodes: $(a),(b)$ - the same experiment, the electrodes are identical graphite rods $\varnothing 15 \mathrm{~mm} ;(c)-(d)$ - massive anode (bowl $\varnothing 150 \mathrm{~mm}$ with a depth of $10 \mathrm{~mm}) ;(c, d)$ - different frames of one experiment; $(e)$-gap $300 \mathrm{~mm}$ 


\title{
Динамика формы электрической дуги и сопутствующих магнитогазодинамических течений, возникающих при размыкании изначально замкнутых электродов
}

\author{
А.П. Глинов, А.П. Головин, П.В. Козлов, К.В. Шалеев \\ НИИ механики МГУ имени М.В. Ломоносова, \\ Россия, г. Москва, 119192, Мичуринский пр-т 1 \\ krestytroitsk@mail.ru
}

\begin{abstract}
Аннотация
Проведены исследования электродугового разряда в воздушной среде атмосферного давления, как между соизмеримыми по размерам электродами, так и на массивных анодах, многократно превышающих по размерам катод. Основной акцент сделан на экспериментальном исследовании вертикальных дуг, инициируемых размыканием электродов, путем проведения высокоскоростной видеорегистрации разряда и синхронного снятия осциллограмм тока и напряжения дугового столба. Показано, что протекание электрического тока и течений межэлектродной среды существенно зависит от скорости размыкания электродов и размеров и формы анодов.
\end{abstract}

Ключевые слова: электродуговой разряд, инициирование дуг, неустойчивость разряда, стабилизация дуги.

\section{1. Введение}

Работа посвящена экспериментальному изучению электродуговых разрядов в открытой воздушной среде между преимущественно графитовыми электродами разной формы и размеров. Исследования проведены на установке П-2000 [1].

Цель проводимых исследований - изучение неустойчивостей разряда и отработка способов их стабилизации, в частности, с помощью наложения внешних магнитных полей, согласования размеров и формы катодного и анодного узлов, и формирования развитых электродных факелов. Для стабилизации разрядной плазмы создан ряд магнитных систем (МС), позволяющих также проводить исследования по устойчивости электродуговых разрядов в магнитном поле [2-4]. Основной акцент сделан на изучении влияния режимов инициирования разряда на формирование и развитие как токового канала, так и сопутствующих ему течений межэлектродной среды. Для достижения возможности получения различных режимов размыкания первоначально сомкнутых электродов была адаптирована разработанная ранее система инициирования дуг [5] путем комбинирования различных противовесов для координатного и блокового механизма электромеханического устройства раздвижки.

Течения межэлектродной среды (в частности, электродные струи-факелы) представляют собой важную часть проявления существенных характерных особенностей именно дуговых разрядов [6-9]. Получение научных данных о таких струях может быть полезно при разработках различного электрофизического оборудования: плазматронов, рельсотронов, мощных электродуговых источников света. 


\section{2. Расчетно-теоретическое моделирование}

Полномасштабное математическое моделирование разрядных электродуговых процессов представляет собой довольно сложную (и пока не решенную задачу) как в постановочном, так и программно-алгоритмическом плане. Обусловлено это тем, что обычно имеют значение процессы, как в самом разрядном канале, так и в электродах и изоляторах и внешней цепи. Наибольшая сложность возникает в построении и замыкании моделей переноса зарядов и нейтральных частиц в пограничных зонах между разнородными средами. И, наконец, возникают при математическом моделировании проблемы доступности (наличия) баз данных, проблемы многих, сильно различающихся, пространственных и временных масштабов. Поэтому исследователи часто прибегают либо к решению модельных задач $[9,10]$ либо проводят экспериментальные исследования [6].

Среди теоретических работ, посвященных изучению гидродинамических течений (без учета протекающих токов) следует отметить работу [11], в которой исследованы автомодельные течения вязкой жидкости между раздвигающимися параллельными пластинами. Математически такие задачи (даже чисто гидродинамические) достаточно сложные. Поэтому нами была предпринята попытка промоделировать влияние раздвижки электродов на стабильность горения протяженных сильноточных дуг не только экспериментально, но и на основе решения задач по электротехнической модели при различных начальных условиях (как минимальных токах зарождения дуги, несколько ампер, так и токах короткого замыкания):

$$
\begin{gathered}
L \frac{\mathrm{d} I}{\mathrm{~d} t}=E-I R-U(X, I), \\
U=a+b X+\frac{c+d X}{I}, \\
X=\left\{(t)= \begin{cases}V_{0} t, & t<t_{0}=\frac{l_{0}}{V_{0}}, \\
l_{0}\left[1+\alpha \exp \left(-\gamma\left(t-t_{0}\right)\right) \sin \omega\left(t-t_{0}\right)\right], & t \geq t_{0}\end{cases} \right.
\end{gathered}
$$

Здесь (1) - уравнение Кирхгофа; (2) - падение напряжения дуги $U$ в межэлектродном промежутке $x$ (уравнение Н. Ayrton [12]); (3) - заданная форма изменения зазора $x$ со временем $t$. Эта форма эмпирическая, была взята на основе обработки экспериментальных данных [1]. Величины $R, I$ и $E$ - балластное сопротивление, разрядный ток и ЭДС источника его питания. Остальные параметры модели: $V_{0}=$ const - скорость раздвижки электродов на требуемое расстояние (зазор) $l_{0} ; a, b, c, d-$ константы H. Ayrton, зависящие от разрядной среды и материалов электродов; $\gamma, \omega$ - декремент и частота колебаний межэлектродного расстояния.

Процессы в исследуемой цепи (1)-(3) представлены на рис. 1. в безразмерных переменных: $x=X / l_{0}-$ зазор; $y=R I / E-$ ток; $u=U / E$ - напряжение; $\tau=V_{0} t / l_{0}$ - время.

Черным цветом выделены кривые базового варианта, отвечающие следующим параметрам:

$$
\begin{gathered}
\pi_{\mathrm{K} 3}=\frac{a}{E}=\pi_{\mathrm{K} 3}^{\sigma}=0.1, \quad \pi_{\mathrm{CT}}=\frac{b l_{0}}{E}=\pi_{\mathrm{CT}}^{\sigma}=0.5, \quad \Gamma=\frac{\gamma l_{0}}{V_{0}}=\Gamma^{\sigma}=1, \quad \Omega=\frac{\omega l_{0}}{V_{0}}=\Omega^{\sigma}=5, \\
s=\frac{c R}{E^{2}}=s^{\sigma}=1.7 \times 10^{-4}, \quad \delta=\frac{d l_{0}}{c}=\delta^{\sigma}=60, \quad \beta=\frac{l_{0} R / L}{V_{0}}=\beta^{\sigma}=2 \times 10^{5}, \quad \alpha=\alpha^{\sigma}=0.5
\end{gathered}
$$

Межэлектродный зазор $(x)$, ток $(y)$ и напряжение $(u)$ даны штрих-пунктирными, сплошными и штриховыми кривыми соответственно.

Видно, что при заданных на рис. 1 границах изменений параметров влияние вариаций ЭДС и амплитуды колебаний зазора имеют ключевое значение на стабильность горения дуги. 
В частности, вариант, отвечающий синим кривым показывает пример ее самогашения. Проводились также расчеты и с изменением скорости раздвижки, характеризуемым вариациями параметра $\beta$ от 0.1 до $10 \times \beta^{\sigma}$. Такие изменения не приводили к режимам гашения дуг.
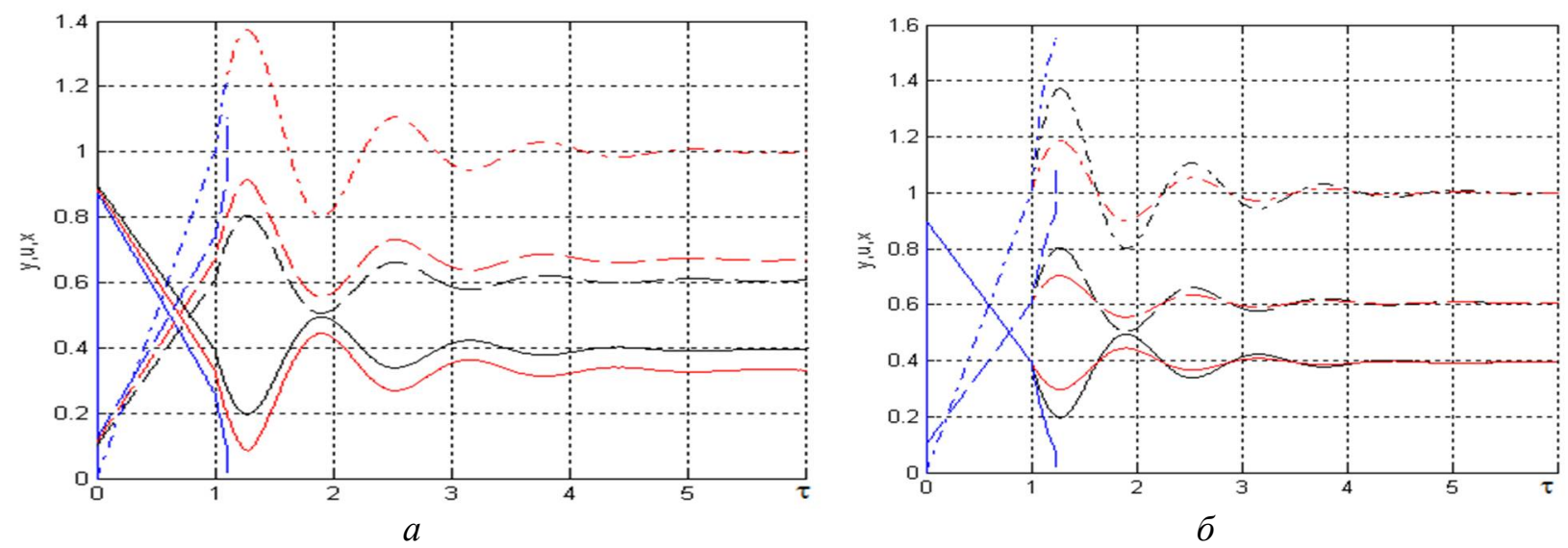

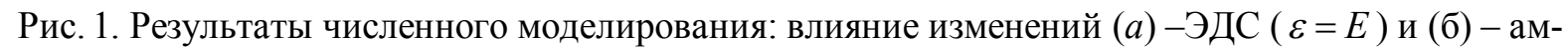
плитуды $(\alpha)$. При изменении цвета (черный, красный и синий) в $(a) \varepsilon=\left(\varepsilon^{\sigma}, \varepsilon^{\sigma} / 1.1, \varepsilon^{\sigma} / 1.1^{2}\right)$ и в (б) $\alpha=\left(\alpha^{6}, 0.5 \alpha^{6}, 1.5 \alpha^{\sigma}\right)$ соответственно

\section{3. Постановка задачи и схема измерений}

Одной из технических проблем при экспериментальном изучении протяженных (несколько см и более) дуг является проблема стабильного инициирования разряда и обеспечение минимальных помех на измерительные цепи. В частности, при инициировании разряда в воздушной среде атмосферного давления при межэлектродных расстояниях $15 \div 18$ мм требовались напряжения осциллятора более 50 кВ [5]. Поэтому в последние годы при изучении протяженных электрических дуг на электроразрядной установке НИИ механики МГУ применяется инициация разряда посредством смыкания электродов с последующим их раздвижением до выбранного межэлектродного расстояния $l_{0}=l_{\text {мэ}}$. Характерное время раздвижки электродов составляет в экспериментах $0.1 \div 0.2$ с. Общая схема регистрации видеоизображений разряда и температуры электродов представлена на рис. 2. Синхронно снимались и осциллограммы тока $I(t)$ и напряжения $U(t)$ (где $t$ - время) на разрядном промежутке. Проведено две серии измерений: первая - с анодами с соизмеримыми катоду размерами $\varnothing=16$ мм; вторая - с массивными анодами, многократно превосходящими по размерам катод. Материал электродов графит 3ОПГ.

Зеркала (3) справа и слева предназначены для получения в кадрах трех проекций изображений, что дает представление о пространственной картине разряда (панорамная видеосъемка). Изучается протяженный электродуговой разряд с квазистационарными токами в воздушной среде атмосферного давления на графитовых электродах и при наличии внешнего магнитного поля, обеспечиваемого той или иной магнитной системой (МС).

Проводимые модельные эксперименты дают информацию о структуре плазменных якорей, важным элементом различных электрофизических установок, в частности, МГД-генераторов, электродуговых ускорителей, электродинамических ускорителей макротел с плазменным якорем, плазмотронов. Эффективность и ресурс работы подобных установок существенно зависит от компактности и степени пространственной однородности таких якорей.

Основное внимание уделено визуализации процессов, связанных с протеканием тока с помощью высокоскоростной $(1200 \div 60000$ кадр/с) съемки в видимом диапазоне длин волн $(0.4 \div 0.8$ мкм). Дополнительно синхронно проводятся пирометрические измерения температуры на поверхности электродов, и регистрируются данные измерений разрядного тока и напряжения на разрядном промежутке. 


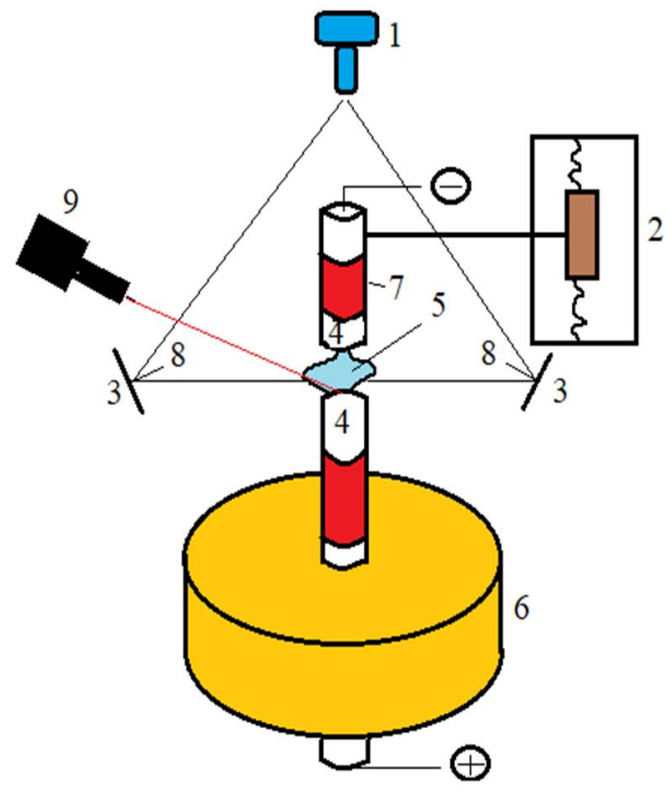

Рис. 2. Схема установки: 1 - видеокамера, 2 - механизм раздвижки электродов, 3 - зеркала, 4 - электроды, 5 - дуга, 6 - изоляционная подставка, 7 - крепежные зажимы, 8 - нормали к зеркальным поверхностям, 9 - пирометр

\section{4. Разряды между идентичными стержневыми электродами}

\section{1. Оценки скорости потоков}

Эксперименты с дугами на соизмеримых графитовых стержневых электродах показали, что форма дуги (при квазистационарных режимах ее питания) весьма нестационарная и неединственная [5]. Соответственно и картина течения межэлектродной среды (особенно при размыкании электродов) достаточно сложная, включающая динамику дугового столба, электродных факелов и опорных пятен. Для ее визуализации и проведения оценок скоростей проводились эксперименты с дуговым разрядом на стержневом графитовом аноде, обмазанном с помощью крахмального клея графитовой крошкой $\varnothing \sim 100$ мк (рис. $3, a$ ). Химическая формула крахмала: $\left(\mathrm{C}_{6} \mathrm{H}_{10} \mathrm{O}_{5}\right) x$, где $x$ - непостоянная (ввиду неоднородности) величина в пределах $100 \div 50000$. Такая обмазка анода не привносила дополнительных загрязнений в элементный состав межэлектродной среды разряда в воздухе между графитовыми электродами. Результаты анализа экспериментов при задаваемом межэлектродном зазоре $l_{\text {мэ }}=37$ мм приведены на рис. 3, 6 -г. В данной серии экспериментов удалось обеспечить стабильный режим раздвижения электродов (без резких колебаний зазора $l_{\text {мэ }}$ см. рис. $3, \sigma$, черная кривая). Здесь на кривой зависимости тока от времени (красная линия): $\mathrm{AB}$ - зона короткого замыкания (КЗ) разрядной системы, ВC - выход на заданный зазор, CD - переходная область стабилизации зазора, DE - квазистационарный режим горения разряда, EF - на фоне стабильного зазора возникновение различных неустойчивых конфигураций дуги [5], FG - гашение дуги. Картина выхода частиц с разогретой поверхности анода приведена на рис. 1(в). Анализ динамики таких видеокадров (с учетом панорамных изображений от зеркал 3 , см. рис. 1) с течением времени позволил определить скорости частиц в катодной струе около анодной поверхности (рис. 3, 2). Точность диагностики находится в пределах $2 \div 20 \%$, причем она наихудшая (20\%) при малых скоростях. Здесь P1, P2 - частицы; PL - плюм - струя среды сложного состава из плазмы, газа и частиц (поток так называемых флюидов с анода); SP1-SP3 - анодные пятна. Как видно, диапазон скоростей разрядной среды $5 \div 50 \mathrm{M} / \mathrm{c}( \pm 1 \mathrm{M} / \mathrm{c})$. 


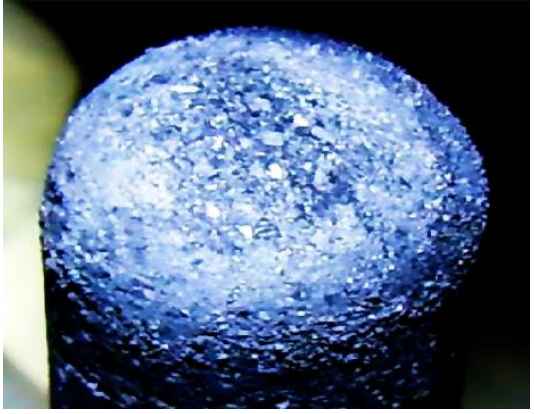

$a$

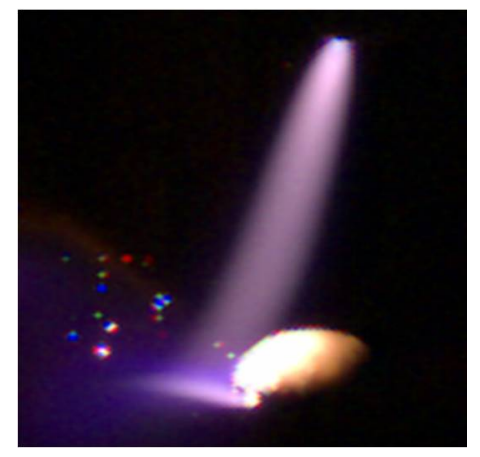

B

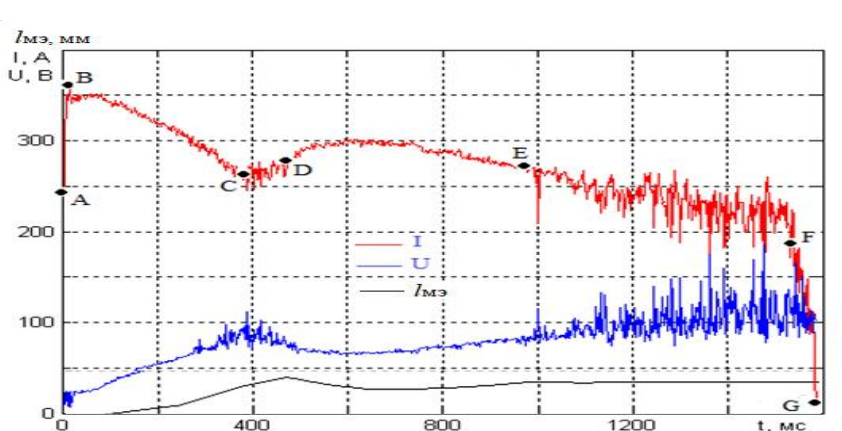

б

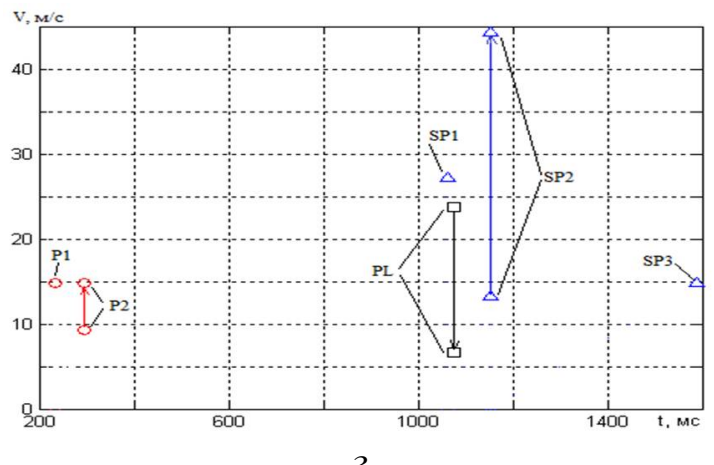

Рис. 3. Эксперименты с обмазанным графитовой крошкой анодом (a): (б) - осциллограммы

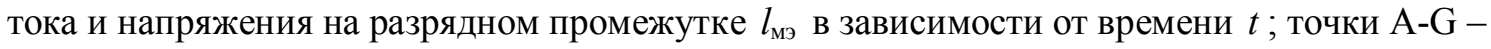
границы различных режимов разряда; (в) - выход плазменного плюма с разогретого анода с выбросом частиц с поверхности; (2) - скорости частиц, сносимых катодной струей около левой поверхности анода $(\mathrm{P} 1,2)$, анодного пятна $(\mathrm{SP} 1,2,3)$ и плюма с поверхности анода $(\mathrm{PL})$, стрелки дают направление движения; скорость регистрации кадров $f_{\mathrm{per}}=12001 \mathrm{\kappa} / \mathrm{c}$

\section{2. Автоколебательный режим}

Обнаружены квазипериодические по времени режимы изменения формы токового канала, обусловленные взаимодействием катодной и анодной струй и эрозионными струями с поверхности разогретого анода (рис. 4).

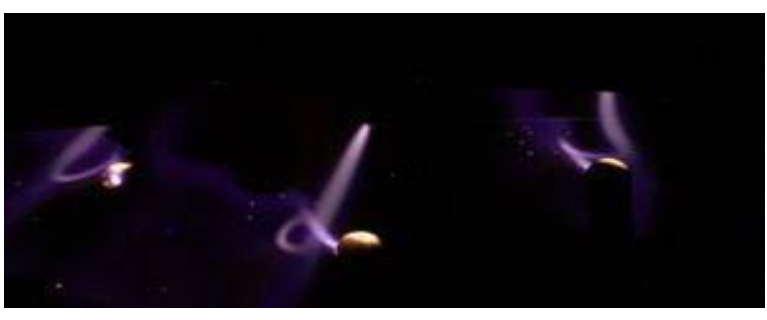

$a$

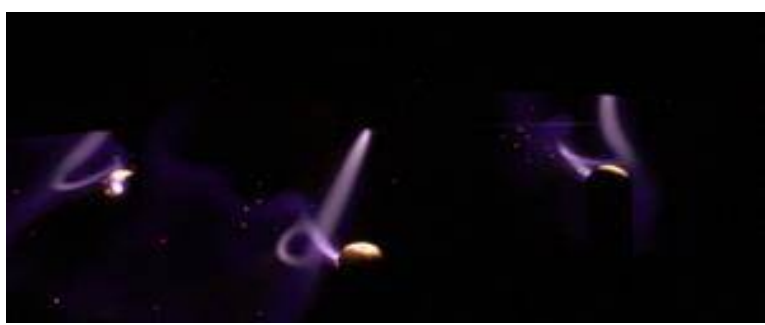

B

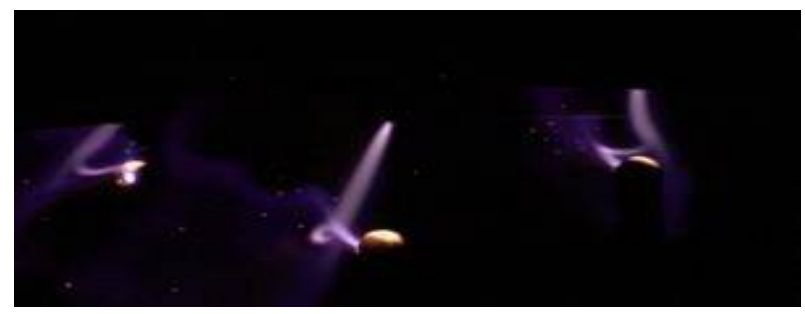

$\sigma$

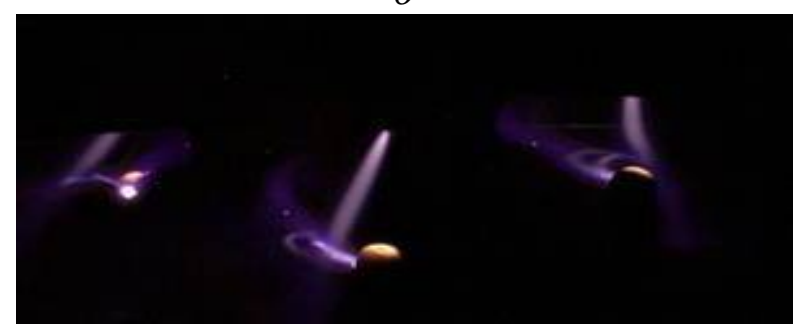

2

Рис. 4. Автоколебательный режим разряда, обусловленный динамикой шунтирующих дуг в прианодной области между электродными факелами: $f_{\text {рег }}=12001 \kappa / c ; l_{\text {мэ }}=37$ мм. Петли тока большие $(a, b)$, малые $(\sigma, 2) ;(2)$ - правый кадр(зеркало) - шунтирующие дуги 


\section{3. Влияние режимов раздвижки}

Также при $l_{\text {мэ }}=37$ мм были проведены эксперименты и при немонотонных режимах раздвижки электродов, что обеспечивалось недостаточным уравновешиванием раздвижных узлов (рис. 5, кривые 2 на $(a, \sigma))$. Уравновешенное раздвижение электродов (кривые 1) позволяло в сопоставимых режимах (кривые 2) получить стабильный (рис. 5,8$)$ разряд по сравнению с дугой при сильно немонотонной раздвижке (рис. 5,2$)$.

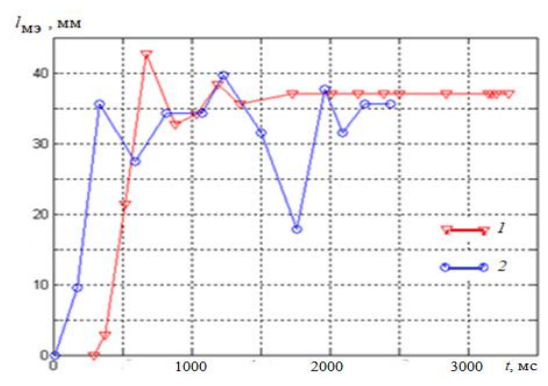

$a$

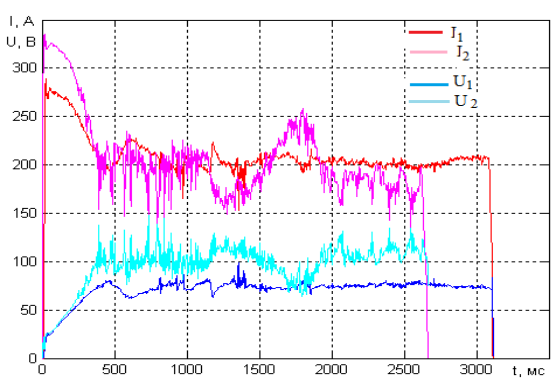

б

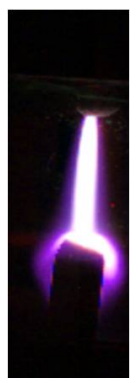

B

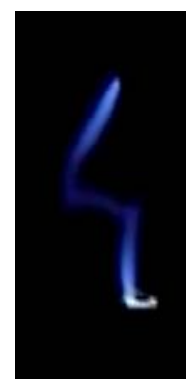

2

Рис. 5. Влияние стабильности процесса раздвижки электродов на устойчивость разряда: $a-$ законы раздвижения электродов $l_{\text {мэ }}(t)$; режим штатный (1) и - нестабильный (2) (кривая 1 смещена от начального времени для улучшения восприятия изображения); $\sigma$ - осциллограммы токов и напряжений дуги; 6,2 - видеокадры разряда при штатном и нештатном размыкании электродов: $f_{\text {per }}=12001 \kappa / \mathrm{c}(a), 1200 \kappa / \mathrm{c}(б)$

Оказалось, что традиционные способы стабилизации дуг как с помощью аксиального внешнего магнитного поля соленоида, так и квазиазимутального поля стержневой МС работают при умеренных полях, но при превышении магнитным полем критической величины развивается винтовая неустойчивость разряда [3]. Использование рассмотренных МС позволило получить стабильное горение открытых вертикальных воздушных дуг до межэлектродных зазоров порядка $15 \mathrm{~cm}$.

\section{5. О возможности стабилизации дуг на массивных анодах}

Проведены эксперименты протяженного дугового разряда на массивных анодах при различных скоростях раздвижения электродов (рис. 6). Рассмотрены два регулярных режима раздвижки электродов: достаточно быстрый $(a, 2)$ и медленный $(\sigma, \varepsilon)$ режим.
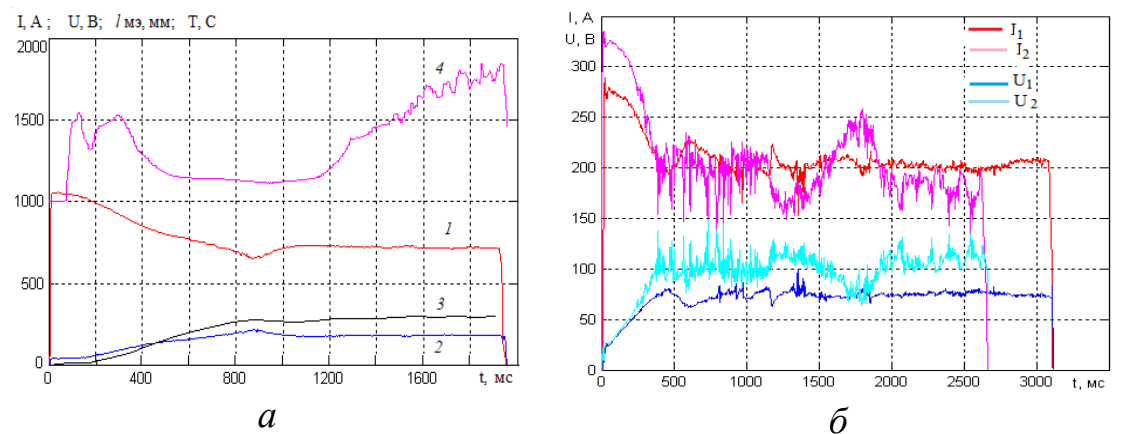

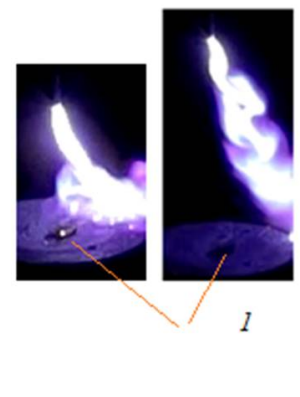

B

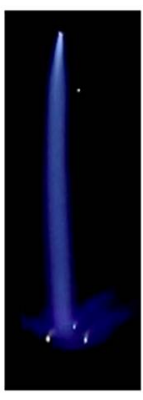

2

Рис. 6. Влияние скорости размыкания электродов на стабильность разряда: $a, \sigma$ - осциллограммы тока (1), напряжения (2), межэлектродного зазора (3) и температуры анода (4); в, 2 - типичные видеокадры разряда на массивном аноде при несколько заторможенном (в) и достаточно быстром (2) размыкании электродов; номер кадра и межэлектродный зазор - 3794 и 300 мм (2), 1587, 1825 и 112 и 212 мм (в) соответственно, 1 - зона наибольшего разрушения анода из-за задержки размыкания электродов; $f_{\text {рег }}=1200 \kappa / c ;$ анод - массивный в виде чаши $(\varnothing 150$ мм); П869( $a, 2), \Pi 870(\sigma, \varepsilon)$ 
Первый из них $(a, 2)$ позволил получить стабильный разряд на зазоре в 30 см. Этот размер равен удвоенному диаметру анода. Если применить результаты анализа винтовой неустойчивости по модели дуги в трубке [9], то двойной диаметр соответствует периоду винтовой структуры. Тогда с увеличением зазора (без увеличения) размера анода последовала бы винтовая неустойчивость. Однако пока наш механизм раздвижки электродов не позволяет получать стабильно выход на большие расстояния и нуждается в модернизации. Второй (б, в) режим привел к интенсивной эрозии анода, загрязнению и охлаждению разрядной плазмы с развитием неоднородных плазменных структур, погасившим дугу.

\section{6. Заключение}

Показано, что протекание электрического тока и течений межэлектродной среды существенно зависит от скорости размыкания электродов и размеров и формы анодов. На массивных анодах изучены протяженные квазистационарные дуги и найдены условия их устойчивого горения. В дугах (между соизмеримыми стержневыми электродами) исследованы квазипериодические по времени режимы изменения формы токового канала, обусловленные взаимодействием катодной и анодной струй и эрозионными струями с поверхности разогретого анода. Проведены расчетно-теоретические моделирования дуговых процессов в переменном межэлектродном зазоре, определившие параметры стабильности дуги.

\section{Благодарности и ссылки на гранты}

Авторы признательны профессору Г.А. Любимову за внимание и интерес к работе, выполненной в НИИ механики МГУ имени М.В. Ломоносова (госконтракт: №AAАA-А16116021110198-5) при финансовой поддержке РФФИ (грант: №18-29-21022).

\section{Литература}

1. A P Glinov, A P Golovin, P V Kozlov, K V Shaleev, G A Lyubimov, «Study of arc discharges on the P-2000 facility» J. Phys.: Conf. Ser. 1250012019.

2. Герман В.О., Глинов А.П., Головин А.П., Козлов П.В. // Прикладная физика. 2015. №5. С. $33-$ 38.

3. Глинов А.П., Головин А.П., Шалеев К.В. // Прикладная физика, 2018. № 2. С. 21-28.

4. Герман В.О., Глинов А.П., Головин А.П., Козлов П.В., Шалеев К.В. // Прикладная физика, 2016. № 6. C.18-24.

5. German V.O., Glinov A.P., Golovin A.P., Kozlov P.V., and Lyubimov G.A. // Plasma Physics Reports, 2013. Vol. 39. No. 13. Pp.1142-1148.

6. Брон О.Б., Сушков Л.К. Потоки плазмы в электрической дуге выключающих аппаратов. Л.: Энергия, 1975 г. $212 \mathrm{c}$.

7. Богомаз А.А., Будин А.В., Коликов В.А., Пинчук М.Э., Позубенков А.А., Рутберг Ф.Г. Исследование влияния катодной и анодной струй на свойства сильноточной электрической дуги // ЖТФ 2002, т. 72, вып. 1, с. 28-35.

8. Max Karasik, Driven Motion and Instability of an Atmospheric Pressure Arc. // PhD Dissertation. Princeton, 2000.

9. Недоспасов А.В., Хаит В.Д. Основы физики процессов в устройствах с низкотемпературной плазмой. М.: Энергоатомиздат, 1991. 224 с.

10. Жуков М.Ф., Коротеев А.С. Теория термической электродуговой плазмы. Ч. 1, 2. Н.: СО Наука, $1987,576 \mathrm{c}$.

11. Петров А.Г. //Известия РАН МЖГ, 2019. №1. С. 58-67.

12. Ayrton H. The Electric Arc., The Electrician Series, D. Van Nostrand Company, Inc., N.Y., pp. 120, 130, 1902. 